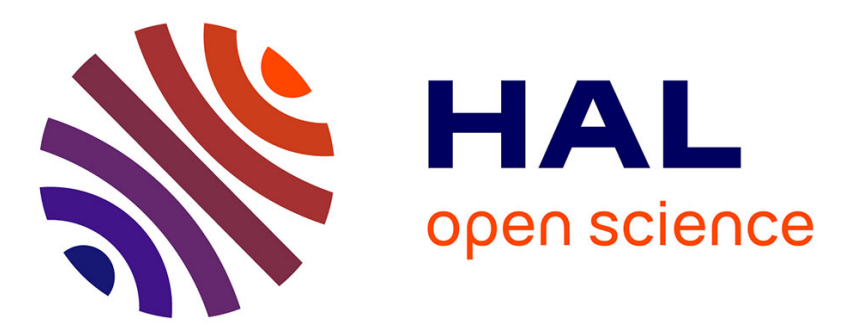

\title{
Analysis of subgrid models of heat convection by symmetry group theory
}

Dina Razafindralandy, Aziz Hamdouni

\section{To cite this version:}

Dina Razafindralandy, Aziz Hamdouni. Analysis of subgrid models of heat convection by symmetry group theory. Comptes Rendus Mécanique, 2007, 335 (n.a.), pp.225-230. hal-00312283

\section{HAL Id: hal-00312283 \\ https://hal.science/hal-00312283}

Submitted on 1 Apr 2019

HAL is a multi-disciplinary open access archive for the deposit and dissemination of scientific research documents, whether they are published or not. The documents may come from teaching and research institutions in France or abroad, or from public or private research centers.
L'archive ouverte pluridisciplinaire HAL, est destinée au dépôt et à la diffusion de documents scientifiques de niveau recherche, publiés ou non, émanant des établissements d'enseignement et de recherche français ou étrangers, des laboratoires publics ou privés. 


\title{
Analysis of subgrid models of the heat convection by symmetry group theory
}

\author{
Dina Razafindralandy, Aziz Hamdouni \\ LEPTAB - Université de La Rochelle \\ Avenue Michel Crépeau, 17042 La Rochelle Cedex 01, France
}

\begin{abstract}
Symmetries, i.e transformations which leave the set of the solutions of the equations unchanged, play an important role in turbulence (conservation laws, wall laws, ...). They should not be destroyed by turbulence models. The symmetries of the heat convection equations are then presented, for a non-isothermal fluid. Next, the subgrid stress tensor and flux models are analyzed, using the symmetry approach.
\end{abstract}

Résumé

Analyse de modèles de sous-maille de la convection thermique par les symétries

Les symétries, c'est-à-dire les transformations laissant invariant l'ensemble des solutions des équations, jouent un rôle important dans la turbulence (lois de conservation, lois de paroi, ...). Ils ne devraient pas être détruits par l'introduction des modèles de turbulence dans les équations. Dans ce papier, on analyse l'invariance des modèles se sous-maille pour la convection thermique sous l'action du groupe de symétrie des équations non filtrées.

Key words: Turbulence; Heat convection ; Subgrid models ; Symmetry group

Mots-clés : Turbulence; Convection thermique; Modèles de sous-maille; Groupe de symétrie

\section{Version française abrégée}

Considérons un fluide newtonien incompressible, de densité $\rho$, de viscosité cinématique $\nu$, de coefficient d'expansion thermique $\beta$ et de diffusivité thermique $\kappa$. Le mouvement de ce fluide est régi par les équations de la convection thermique (1), où $g$ est l'accélération de la pesanteur et $\mathbf{e}_{3}$ le vecteur unitaire vertical ascendant. Ces équations possèdent des transformations, appelées symétries, qui, à une solution, font correspondre une autre solution. Ces transformations ont un rôle important dans la turbulence (lois de conservation, lois de paroi, ...). Il est donc essentiel que les modèles de turbulence ne les détruisent pas.

Dans le cas d'un fluide isotherme, Oberlack ([1]) analysait des modèles de sous-maille sous l'angle de l'invariance par les symétries des équations de Navier-Stokes. Cette étude a été étendue par Razafindralandy ([2]) et Razafindralandy et Hamdouni ([3]) à une classe plus large de modèles de sous-maille. Il a alors été montré que très peu de modèles respectent les symétries. Dans [4], Razafindralandy et Hamdouni ont ainsi proposé une classe de modèles compatibles avec les symétries des équations et ont obtenu de meilleurs résultats par rapport au modèle de Smagorinsky et au modèle dynamique pour la configuration d'une cavité ventilée.

Dans ce papier, on se propose d'étendre l'analyse au cas des écoulements anisothermes. Dans un premier temps, dans la section 2, nous donnons le groupe de symétrie des équations de la convection thermique. Ce sont les transformations (2)-(9). Nous présentons ensuite dans la section 3 les principaux modèles de sous-maille "usuels", qui seront analysés dans la section 4. L'analyse de l'invariance des modèles par le groupe de symétrie des équations (1) conduit aux résultats suivants : tous les modèles sont invariants par

Email addresses: drazafin@univ-lr.fr (Dina Razafindralandy), ahamdoun@univ-lr.fr (Aziz Hamdouni).

Preprint submitted to 
les symétries (2)-(6); en revanche, seuls le modèle dynamique et le modèle de similarité d'échelle sont invariants sous les transformations d'échelle (7) et (8) (voir tableau 1), pourtant ces transformations ont un rôle particulier par exemple dans l'élaboration des lois d'échelles dans [5]. De plus, les deux modèles qui respectent toutes les symétries des équations font intervenir un filtre test qui est susceptible de détruire les propriétés d'invariance. Cela nous a amené à construire d'autres types de modèles préservant les symétries des équations de la convection thermique qui seront présentés dans un futur papier.

\section{Introduction}

A turbulent fluid flow has natural and fundamental properties, such as the invariance of the motion equations under the action of their symmetry group. The symmetries of an equation are transformations which take a solution into another solution. They play an important role in turbulence (conservation laws [6], wall laws [7], spectrum form [8], exact solutions [9], ...). In some extent, they contains the physics of the equations. Though, Oberlack $([1])$, and next Razafindralandy and Hamdouni $([2,10])$ showed that many subgrid stress models violate the symmetry group of Navier-Stokes equations, in the case of an isothermal fluid. This observation led the last authors to propose a new class of subgrid models which respect the symmetries of Navier-Stokes equations and then preserve the physical properties of the flow $([11,3])$. In addition, a test on a simple model of the class gave results which was better than those provided by Smagorinsky and the dynamic models, for ventilated cavity flow.

In this article, we will present the symmetry group of the heat convection equations for non-isothermal fluid flows and analyze the common turbulent subgrid stress tensor and flux models. This analysis is necessary before deciding if a development of symmetry consistent models is needed. The paper will be organized as follows. In section 2 , the symmetry group of the heat convection equations will be presented. In section 3, a recall of the common subgrid models will be done. These models will be analyzed using the symmetry group of the equations in section 4 .

\section{The symmetry group of the heat convection equations}

Consider an incompressible Newtonian fluid with a density $\rho$, kinematic viscosity $\nu$, thermal expansion coefficient $\beta$ and thermal diffusivity $\kappa$. Let $g$ be the gravity acceleration and $\mathbf{e}_{3}$ the ascending vertical unitary vector. If $t$ is the time variable, $\mathbf{x}=\left(x_{i}\right)_{i}$ the space variable, $\mathbf{u}$ the velocity field, $p$ the pressure and $\theta$ the temperature then the motion of this fluid is governed by the heat convection equations:

$$
\left\{\begin{array}{l}
\operatorname{div} \mathbf{u}=0, \quad \frac{\partial \mathbf{u}}{\partial t}+\operatorname{div}(\mathbf{u} \otimes \mathbf{u})+\frac{1}{\rho} \nabla p-\operatorname{div} \boldsymbol{T}_{r}-\beta g \theta \mathbf{e}_{3}=0 \\
\frac{\partial \theta}{\partial t}+\operatorname{div}(\theta \mathbf{u})-\operatorname{div} \mathbf{h}_{r}=0
\end{array}\right.
$$

In these expressions, $\boldsymbol{T}_{r}=2 \nu \boldsymbol{S}$ is the viscous stress tensor, $\boldsymbol{S}=\left[\nabla \mathbf{u}+{ }^{T} \nabla \mathbf{u}\right] / 2$ the strain rate tensor and $\mathbf{h}_{r}=\kappa \nabla \theta$ the heat flux.

Let $\mathbf{y}=(t, \mathbf{x}, \mathbf{u}, p)$. A one-parameter transformation of (1) is a function

$$
T_{a}: \mathbf{y} \mapsto \widehat{\mathbf{y}}=\widehat{\mathbf{y}}(\mathbf{y}, a)
$$

which depends continuously on the real parameter $a . T_{a}$ is a symmetry of (1) if it leaves the set of solutions of (1) unchanged. Thanks to Lie's theory ([12]), all the one-parameter symmetries of the heat convection equations can be computed. They are generated by the following transformations:

- the time translation:

- the pressure translation:

$$
(t, \mathbf{x}, \mathbf{u}, p, \theta) \longmapsto(t+a, \mathbf{x}, \mathbf{u}, p, \theta)
$$

$$
(t, \mathbf{x}, \mathbf{u}, p, \theta) \longmapsto(t, \mathbf{x}, \mathbf{u}, p+\zeta(t), \theta),
$$

- the pressure-temperature translation:

$$
(t, \mathbf{x}, \mathbf{u}, p, \theta) \longmapsto\left(t, \mathbf{x}, \mathbf{u}, p+a \beta g x_{3}, \theta+a / \rho\right),
$$


- the horizontal rotation:

$$
(t, \mathbf{x}, \mathbf{u}, p, \theta) \longmapsto(t, \boldsymbol{R} \mathbf{x}, \boldsymbol{R u}, p, \theta),
$$

- the generalized Galilean transformation:

$$
(t, \mathbf{x}, \mathbf{u}, p, \theta) \longmapsto(t, \mathbf{x}+\boldsymbol{\alpha}(t), \mathbf{u}+\dot{\boldsymbol{\alpha}}(t), p+\rho \mathbf{x} \cdot \ddot{\boldsymbol{\alpha}}(t), \theta),
$$

the space translation corresponding to the case where the function $\boldsymbol{\alpha}=\left(\alpha_{i}\right)_{i}$ is constant and the classical Galilean transformation to the case where $\boldsymbol{\alpha}$ is linear,

- and the first scaling transformation:

$$
(t, \mathbf{x}, \mathbf{u}, p, \theta) \longmapsto\left(\mathrm{e}^{2 a} t, \mathrm{e}^{a} \mathbf{x}, \mathrm{e}^{-a} \mathbf{u}, \mathrm{e}^{-2 a} p, \mathrm{e}^{-3 a} \theta\right)
$$

which shows how $\mathbf{u}, p$ and $\theta$ change when the spatio-temporal scale is multiplied by $\left(\mathrm{e}^{a}, \mathrm{e}^{2 a}\right)$.

In these expressions, $\zeta$ and $\boldsymbol{\alpha}$ are arbitrary functions of time and $\boldsymbol{R}$ a constant 2D horizontal rotation matrix. In addition to these symmetries, one can also consider one-parameter symmetries of the form

$$
(t, \mathbf{x}, \mathbf{u}, p, \theta, \nu, \kappa) \longmapsto(\widehat{t}, \widehat{\mathbf{x}}, \widehat{\mathbf{u}}, \widehat{p}, \widehat{\theta}, \widehat{\nu}, \widehat{\kappa}) .
$$

Such a symmetry, called equivalence transformation, takes a solution of (1) into a solution of other heat convection equations, with a different value of $\nu$ and $\kappa$. Again using Lie's theory, we obtain an equivalence transformation of (1), the second scaling transformation:

$$
(t, \mathbf{x}, \mathbf{u}, p, \theta, \nu, \kappa) \longmapsto\left(t, \mathrm{e}^{a} \mathbf{x}, \mathrm{e}^{a} \mathbf{u}, \mathrm{e}^{2 a} p, \mathrm{e}^{a} \theta, \mathrm{e}^{2 a} \nu, \mathrm{e}^{2 a} \kappa\right)
$$

which shows the consequence of a change of the spatial scale.

Another known symmetry of the heat convection equations is the reflection:

$$
(t, \mathbf{x}, \mathbf{u}, p, \theta) \mapsto\left(t, \mathbf{\Lambda} \mathbf{x}, \mathbf{\Lambda} \mathbf{u}, p, \iota_{3} \theta\right)
$$

where $\boldsymbol{\Lambda}$ is the diagonal matrix $\boldsymbol{\Lambda}=\operatorname{diag}\left(\iota_{1}, \iota_{2}, \iota_{3}\right)$ and $\iota_{i}= \pm 1, i=1,2,3$. The reflection is not a one-parameter transformation and could not thus be computed like the previous symmetries.

The symmetry group of (1) is the group spanned by all the symmetries (2)-(9). This group will be used to analyze the common subgrid models.

\section{Common subgrid models}

After filtering, the large-eddy simulation method gives

$$
\left\{\begin{array}{l}
\operatorname{div} \overline{\mathbf{u}}=0, \\
\frac{\partial \bar{\theta}}{\partial t}+\operatorname{div}(\bar{\theta} \overline{\mathbf{u}})-\operatorname{div}\left(\overline{\mathbf{h}}_{r}-\mathbf{h}\right)=0 .
\end{array}\right.
$$

The symbol bar $\left(^{-}\right)$stands for filtering. $\boldsymbol{T}=\overline{\mathbf{u} \otimes \mathbf{u}}-\overline{\mathbf{u}} \otimes \overline{\mathbf{u}}$ is the subgrid stress tensor and $\mathbf{h}=\overline{\theta \mathbf{u}}-\bar{\theta} \overline{\mathbf{u}}$ the subgrid flux. These two terms must be modeled in order to close the equations.

- The most commonly used model is the Smagorinsky model which was derived by adopting the concept of turbulent viscosity for $\boldsymbol{T}$ and an analogy for $\mathbf{h}$ :

$$
\boldsymbol{T}^{d}=-2\left(C_{s} \bar{\delta}\right)^{2}|\overline{\boldsymbol{S}}| \overline{\boldsymbol{S}}, \quad \mathbf{h}=-\frac{\left(C_{s} \bar{\delta}\right)^{2}}{P r_{s g}}|\overline{\boldsymbol{S}}| \nabla \bar{\theta},
$$

with $|\overline{\boldsymbol{S}}|^{2}=2 \sum_{i j} \overline{\boldsymbol{S}}_{i j}^{2} . C_{s}$ is the Smagorinsky constant and $\operatorname{Pr}_{s g}$ the subgrid Prandtl number. The superscript $d$ stands for the deviatoric part, i.e. $\boldsymbol{T}^{d}=\boldsymbol{T}-\left(\frac{1}{3} \operatorname{tr} \boldsymbol{T}\right) \boldsymbol{I}_{d}, \boldsymbol{I}_{d}$ being the identity matrix.

- By using Germano-Lilly procedure ([13]), the model constants can be calculated in a dynamic way. This leads to the dynamic model:

$$
\boldsymbol{T}^{d}=-2 C_{\boldsymbol{T}} \bar{\delta}^{2}|\overline{\boldsymbol{S}}| \overline{\boldsymbol{S}}, \quad \mathbf{h}=-C_{\mathbf{h}} \bar{\delta}^{2}|\overline{\boldsymbol{S}}| \nabla \bar{\theta}
$$


where $C_{\boldsymbol{T}}=\operatorname{tr}(\boldsymbol{L} \boldsymbol{M}) / \operatorname{tr}\left(\boldsymbol{M}^{2}\right), C_{\mathbf{h}}=\operatorname{tr}\left(\mathbf{L}^{\prime}{ }^{\mathrm{T}} \mathbf{M}^{\prime}\right) / \operatorname{tr}\left(\mathbf{M}^{\prime}{ }^{\mathrm{T}} \mathbf{M}^{\prime}\right)$ and

$$
\boldsymbol{L}=\widetilde{\overline{\mathbf{u}} \otimes \overline{\mathbf{u}}}-\widetilde{\overline{\mathbf{u}}} \otimes \widetilde{\mathbf{u}}, \quad \boldsymbol{M}=\widetilde{\bar{\delta}}^{2}|\widetilde{\overline{\boldsymbol{S}}}| \widetilde{\overline{\boldsymbol{S}}}-\bar{\delta}^{2} \mid \widetilde{\overline{\boldsymbol{S}} \mid \overline{\boldsymbol{S}}}, \quad \mathbf{L}^{\prime}=\widetilde{\bar{\theta} \overline{\mathbf{u}}}-\widetilde{\bar{\theta}} \widetilde{\overline{\mathbf{u}}}, \quad \mathbf{M}^{\prime}=\bar{\delta}^{2} \widetilde{\overline{\boldsymbol{S}} \nabla \bar{\theta}}-\widetilde{\bar{\delta}} \widetilde{\overline{\boldsymbol{S}}} \widetilde{\bar{\theta}}
$$

The tilde symbolizes a test filtering, with a width $\widetilde{\bar{\delta}}$.

- Another model, which introduces the buoyancy term, is the Eidson model ([14]):

$$
\boldsymbol{T}^{d}=-2 C_{E} \bar{\delta}^{2} \sqrt{|\overline{\boldsymbol{S}}|^{2}-\frac{\beta g}{P r_{s g}} \frac{\partial \bar{\theta}}{\partial x_{3}}} \overline{\boldsymbol{S}}, \quad \mathbf{h}=-\frac{C_{E} \bar{\delta}^{2}}{P r_{s g}} \sqrt{|\overline{\boldsymbol{S}}|^{2}-\frac{\beta g}{P r_{s g}} \frac{\partial \bar{\theta}}{\partial x_{3}}} \nabla \bar{\theta},
$$

where $C_{E}$ is a constant. In practice, when the term under the radical sign is negative, the model is put to zero.

- To avoid the zero setting, Peng and Davidson ([15]) propose a modified version of Eidson model:

$$
\boldsymbol{T}^{d}=-2 C_{E} \bar{\delta}^{2} \frac{1}{|\overline{\boldsymbol{S}}|}\left(|\overline{\boldsymbol{S}}|^{2}-\frac{\beta g}{P r_{s g}} \frac{\partial \bar{\theta}}{\partial x_{3}}\right) \overline{\boldsymbol{S}}, \quad \mathbf{h}=-\frac{C_{E} \bar{\delta}^{2}}{P r_{s g}} \frac{1}{|\overline{\boldsymbol{S}}|}\left(|\overline{\boldsymbol{S}}|^{2}-\frac{\beta g}{P r_{s g}} \frac{\partial \bar{\theta}}{\partial x_{3}}\right) \nabla \bar{\theta} .
$$

- The last model which will be considered is the similarity model, adapted from Bardina model to the non-isothermal case:

$$
\boldsymbol{T}^{d}=\widetilde{\overline{\mathbf{u}} \otimes \overline{\mathbf{u}}}-\widetilde{\mathbf{u}} \otimes \widetilde{\mathbf{u}}, \quad \mathbf{h}=\widetilde{\bar{\theta} \overline{\mathbf{u}}}-\widetilde{\bar{\theta}} \widetilde{\mathbf{u}} .
$$

\section{Model analysis}

The set of solutions $(\mathbf{u}, p, \theta)$ of $(1)$ is preserved by each of the symmetries $(2)-(9)$. We then require that so is the set of solutions $(\overline{\mathbf{u}}, \bar{p}, \bar{\theta})$ of $(10)$ too, since $(\overline{\mathbf{u}}, \bar{p}, \bar{\theta})$ is expected to be a good approximation of $(\mathbf{u}, p, \theta)$. More clearly, if a transformation $T:(t, \mathbf{x}, \mathbf{u}, p, \theta) \mapsto(\widehat{t}, \widehat{\mathbf{x}}, \widehat{\mathbf{u}}, \widehat{p}, \widehat{\theta})$ is a symmetry of $(1)$, we require that the model is such that the same transformation, applied to the filtered quantities, $T$ : $(t, \mathbf{x}, \overline{\mathbf{u}}, \bar{p}, \bar{\theta}) \mapsto(\widehat{t}, \widehat{\mathbf{x}}, \widehat{\mathbf{u}}, \widehat{\bar{p}}, \widehat{\bar{\theta}})$, is a symmetry of (10). When it is the case, equations (10) and the model will be said invariant under the symmetry.

\subsection{Time, pressure and Galilean translations}

All the above models are invariant under the time and pressure translations because neither $t$ nor $p$ is explicitly present in their expressions. Next, applied to the filtered variables, the generalized Galilean transformation is:

$$
(t, \mathbf{x}, \overline{\mathbf{u}}, \bar{p}, \bar{\theta}) \mapsto(\widehat{t}, \widehat{\mathbf{x}}, \widehat{\overline{\mathbf{u}}}, \widehat{\bar{p}}, \widehat{\bar{\theta}})=(t, \mathbf{x}+\boldsymbol{\alpha}(t), \overline{\mathbf{u}}+\dot{\boldsymbol{\alpha}}(t), \bar{p}+\rho \mathbf{x} \cdot \ddot{\boldsymbol{\alpha}}(t), \bar{\theta}) .
$$

- Since $\widehat{\nabla \overline{\mathbf{u}}}=\nabla \overline{\mathbf{u}}$ and $\widehat{\bar{\theta}}=\bar{\theta}$, Smagorinsky and Eidson models are invariant under the generalized Galilean transformation.

- For the similarity model, we have:

$$
\begin{aligned}
& \widetilde{\widehat{\hat{\mathbf{u}}} \otimes \widehat{\widehat{\mathbf{u}}}}-\widetilde{\widehat{\mathbf{u}}} \otimes \widetilde{\widehat{\mathbf{u}}}=(\overline{\mathbf{u}}+\widetilde{\boldsymbol{\alpha}}) \otimes(\overline{\mathbf{u}}+\dot{\boldsymbol{\alpha}})-(\widetilde{(\overline{\mathbf{u}}+\dot{\boldsymbol{\alpha}}}) \otimes(\widetilde{\overline{\mathbf{u}}+\dot{\boldsymbol{\alpha}}})=\widetilde{\overline{\mathbf{u}} \otimes \overline{\mathbf{u}}}-\widetilde{\overline{\mathbf{u}}} \otimes \widetilde{\mathbf{u}}, \\
& \widetilde{\bar{\theta}} \widehat{\overline{\mathbf{u}}}-\widetilde{\bar{\theta}} \widetilde{\overline{\mathbf{u}}}=\bar{\theta} \widetilde{(\overline{\mathbf{u}}+\dot{\boldsymbol{\alpha}})}-\widetilde{\bar{\theta}}(\widetilde{\overline{\mathbf{u}}+\dot{\boldsymbol{\alpha}}})=\widetilde{\bar{\theta}} \overline{\mathbf{u}}-\widetilde{\bar{\theta}} \widetilde{\overline{\mathbf{u}}} \text {. }
\end{aligned}
$$

The model is then invariant. Notice that the test filter is assumed not to destroy the invariance properties (see [1]).

- Using the same relations, it is easy to show that $\widehat{\boldsymbol{L}}=\boldsymbol{L}, \widehat{\boldsymbol{M}}=\boldsymbol{M}, \widehat{\mathbf{L}^{\prime}}=\mathbf{L}^{\prime}$ and $\widehat{\mathbf{M}^{\prime}}=\mathbf{M}^{\prime}$. The invariance of the dynamic model follows from these expressions.

In summary, all the models are invariant under the time translation, the pressure translation and generalized Galilean transformations. 


\subsection{Pressure-temperature translation}

The filtered equations (10) are invariant under the pressure-temperature translation if and only if $\widehat{\boldsymbol{T}}=\boldsymbol{T}$ and $\widehat{\mathbf{h}}=\mathbf{h}$.

- Smagorinsky and Eidson models are invariant because $\widehat{\overline{\boldsymbol{S}}}=\overline{\boldsymbol{S}}$ and $\widehat{\bar{\theta}}=\bar{\theta}$.

- Next,

$$
\widetilde{\bar{\theta}} \widehat{\overline{\mathbf{u}}}-\widetilde{\bar{\theta}} \widetilde{\overline{\mathbf{u}}}=(\widetilde{\bar{\theta}} \overline{\mathbf{u}}+a \widetilde{\overline{\mathbf{u}}} / \rho)-(\widetilde{\bar{\theta}} \widetilde{\overline{\mathbf{u}}}+a \widetilde{\overline{\mathbf{u}}} / \rho)=\widetilde{\bar{\theta}} \overline{\mathbf{u}}-\widetilde{\bar{\theta}} \widetilde{\overline{\mathbf{u}}}
$$

The similarity model is then invariant.

- For the dynamic model, $\boldsymbol{L}, \boldsymbol{M}$ and $\mathbf{L}^{\prime}$ are unchanged. And since $\widehat{\nabla \theta}=\nabla \theta, \mathbf{M}^{\prime}$ is also unchanged. The model is invariant.

\subsection{Reflection and rotation}

The rotation, applied to the filtered variables, is

$$
(t, \mathbf{x}, \overline{\mathbf{u}}, \bar{p}, \bar{\theta}) \mapsto(\widehat{t}, \widehat{\mathbf{x}}, \widehat{\overline{\mathbf{u}}}, \widehat{\bar{p}}, \widehat{\bar{\theta}})=(t, \boldsymbol{R} \mathbf{x}, \boldsymbol{R} \overline{\mathbf{u}}, \bar{p}, \bar{\theta}) .
$$

This transformation is a symmetry of (10) if and only if $\widehat{\boldsymbol{T}}=\boldsymbol{R} \boldsymbol{T}{ }^{T} \boldsymbol{R}$ and $\widehat{\mathbf{h}}=\boldsymbol{R h}$.

- It is easy to show that $\widehat{\overline{\boldsymbol{S}}}=\boldsymbol{R} \overline{\boldsymbol{S}}{ }^{T} \boldsymbol{R}$ and $\widehat{\nabla} \hat{\bar{\theta}}=\boldsymbol{R} \nabla \bar{\theta}$. Smagorinsky and Eidson models are then invariant.

- Moreover, $\widehat{\overline{\mathbf{u}}} \otimes \widehat{\overline{\mathbf{u}}}=\boldsymbol{R}(\overline{\mathbf{u}} \otimes \overline{\mathbf{u}}){ }^{T} \boldsymbol{R}$ and $\widehat{\bar{\theta}} \widehat{\overline{\mathbf{u}}}=\boldsymbol{R}(\bar{\theta} \overline{\mathbf{u}})$. This leads to the invariance of the similarity model.

- For the dynamic model, we have

$$
\widehat{\boldsymbol{L}}=\boldsymbol{R} \boldsymbol{L}^{T} \boldsymbol{R}, \quad \widehat{\boldsymbol{M}}=\boldsymbol{R} \boldsymbol{M}^{T} \boldsymbol{R}, \quad \widehat{\mathbf{L}^{\prime}}=\boldsymbol{R} \mathbf{L}^{\prime}, \quad \widehat{\mathbf{M}^{\prime}}=\boldsymbol{R} \mathbf{M}^{\prime} .
$$

Hence, $\widehat{C_{\boldsymbol{T}}}=C_{\boldsymbol{T}}$ and $\widehat{C_{\mathbf{h}}}=C_{\mathbf{h}}$. So, $\widehat{\boldsymbol{T}}=\boldsymbol{T}$ and $\widehat{\mathbf{h}}=\mathbf{h}$; the model is invariant.

All the models are then invariant under the rotation.

Next, equations (10) are invariant under the reflection if and only if $\widehat{\boldsymbol{T}}=\boldsymbol{\Lambda} \boldsymbol{T}{ }^{\mathrm{T}} \boldsymbol{\Lambda}$ and $\widehat{\mathbf{h}}=\iota_{3} \boldsymbol{\Lambda} \mathbf{h}$. Using arguments similar to the previous ones, it can be shown that all the models are also invariant under the reflection.

\subsection{Scaling transformations}

The two scaling transformations can be combined into the following two-parameter transformation:

$$
(t, \mathbf{x}, \mathbf{u}, p, \theta, \nu, \kappa) \mapsto\left(\mathrm{e}^{2 a} t, \mathrm{e}^{b+a} \mathbf{x}, \mathrm{e}^{b-a} \mathbf{u}, \mathrm{e}^{2 b-2 a} p, \mathrm{e}^{b-3 a} \theta, \mathrm{e}^{2 b} \nu, \mathrm{e}^{2 b} \kappa\right) .
$$

The first scaling transformation corresponds to $b=0$ and the second to $a=0$. The equations (10) are invariant if and only if $\widehat{\boldsymbol{T}}=\mathrm{e}^{2 b-2 a} \boldsymbol{T}$ and $\widehat{\mathbf{h}}=\mathrm{e}^{2 b-4 a} \mathbf{h}$.

- Since $\widehat{\overline{\boldsymbol{S}}}=\mathrm{e}^{-2 a} \overline{\boldsymbol{S}}$ and $\widehat{\nabla \bar{\theta}}=\mathrm{e}^{-4 a} \nabla \bar{\theta}$, we have, for Smagorinsky model $\widehat{\boldsymbol{T}}=\mathrm{e}^{-4 a} \boldsymbol{T}$ and $\widehat{\mathbf{h}}=\mathrm{e}^{-6 a} \mathbf{h}$. The model verifies the required conditions neither when $a=0$ nor when $b=0$. Thus, it is not invariant either under the first or under the second scaling transformation.

- The first terms of Eidson models are similar to Smagorinsky model. These models are not invariant.

- The similarity model is invariant because

$$
\widetilde{\widehat{\hat{\mathbf{u}}} \otimes \widehat{\hat{\mathbf{u}}}}-\widetilde{\widehat{\mathbf{u}}} \otimes \widetilde{\widehat{\mathbf{u}}}=\mathrm{e}^{2 b-2 a}(\widetilde{\widetilde{\mathbf{u}} \otimes \overline{\mathbf{u}}}-\widetilde{\overline{\mathbf{u}}} \otimes \widetilde{\mathbf{u}}) \quad \text { and } \quad \widetilde{\bar{\theta}} \widehat{\overline{\mathbf{u}}}-\widetilde{\bar{\theta}} \widetilde{\widehat{\mathbf{u}}}=\mathrm{e}^{2 b-4 a}(\widetilde{\bar{\theta} \overline{\mathbf{u}}}-\widetilde{\bar{\theta}} \widetilde{\mathbf{u}})
$$

- For the dynamic model, $\widehat{C_{\boldsymbol{T}}}=\mathrm{e}^{2 b+2 a} C_{\boldsymbol{T}}, \widehat{C_{\mathbf{h}}}=\mathrm{e}^{2 b+2 a} C_{\mathbf{h}}$. This leads to the invariance of the model. In summary, only the dynamic and the similarity models are invariant under the scaling transformations. The non-invariance under these symmetries is very prejudicial for the models. Indeed, these symmetries have a particular role for example in the deduction of wall laws ([5]) and in spectral analysis ([8]). 


\section{Conclusion}

The analysis result is summarized in table 1. From it, we can draw the same conclusion as in the case of Navier-Stokes equations. Only very few models are invariant under the symmetry group of the equations, namely the dynamic and the similarity models. In addition, these two models need a test filter, and the test filter may destroy the invariance properties. In a future paper, new invariant models will be proposed, using the same methodology as in ([11]). Such models preserve the physical properties contained in the symmetries of the equations.

\section{References}

[1] M. Oberlack. Invariant modeling in large-eddy simulation of turbulence. In Annual Research Briefs. Stanford University, 1997.

[2] D. Razafindralandy. Contribution à l'étude mathématique et numérique de la simulation des grandes échelles. Phd, Université de La Rochelle, 2005.

[3] D. Razafindralandy and A. Hamdouni. Analysis and development of subgrid turbulence models preserving the symmetry properties of navier-stokes equations. Submitted to J. of Fluid Mech., 2006.

[4] D. Razafindralandy and A. Hamdouni. Subgrid models preserving the symmetry group of Navier-Stokes equations. C. R. Mécanique, 333:481-486, 2005.

[5] M. Oberlack. Symmetries of the Navier-Stokes equations and their applications for subgrid-models in large eddy simulation of turbulence. In A. Gyr, W. Kinzelbach, and A. Tsinober, editors, Fundamental Problematic Issues in Turbulence. Birkhäuser Verlag, 1999.

[6] E. Nœther. Invariante Variationsprobleme. In Königliche Gesellschaft der Wissenschaften, 235-257, Göttingen, 1918.

[7] M. Oberlack. Symmetries, invariance and scaling-laws in inhomogeneous turbulent shear flows. Flow Turb. and Comb., 62(2):111-135, 1999.

[8] G. Ünal. Application of equivalence transformations to inertial subrange of turbulence. Lie Group Appl., 1(1):232-240, 1994.

[9] W. Fushchych and R. Popowych. Symmetry reduction and exact solutions of the Navier-Stokes equations I. J. Nonlin. Math. Phys., 1(1):75-113, 1994.

[10] D. Razafindralandy and A. Hamdouni. Symmetry invariant subgrid models. In: Guerts, Métais, Lamballais, Friedrich (Eds), Direct and Large-Eddy Simulation 6, Springer Science, France, 2006.

[11] D. Razafindralandy, A. Hamdouni, and C. Béghein. A class of subgrid-scale models preserving the symmetry group of Navier-Stokes equations. To appear in Comm. in Nonlin. Science and Num. Simulation, 2005.

[12] P. Olver. Applications of Lie groups to differential equations. Graduate texts in mathematics. Springer-Verlag, New-York, 1986.

[13] D. Lilly. A proposed modification of the Germano subgrid-scale closure method. Phys. Fluids, A4(3):633-635, 1992.

[14] T. Eidson. Numerical simulation of the turbulent Rayleigh-Bénard problem using subgrid modelling. J. Fluid Mech., 158:245-268, 1985.

[15] S-H. Peng and L. Davidson. Comparison of subgrid-scale models in LES for turbulent convection flow with heat transfer. In: 2nd EF Conference in Turbulent Heat Transfer, 1: 5.25-5.35, Manchester, United Kingdom, 1998.

\begin{tabular}{lccc}
\hline & $\begin{array}{c}\text { Time, pressure, } \\
\text { Galilean }\end{array}$ & Pressure- & Rotation, \\
& temperature & reflection \\
\hline Smagorinsky model & invariant & invariant & invariant \\
Dynamic model* & invariant & invariant & invariant non-invariant \\
Eidson model & invariant & invariant & invariant invariant \\
Modified Eidson model & invariant & invariant & invariant non-invariant \\
Similarity model & invariant & invariant & invariant \\
\hline
\end{tabular}

Table 1

Result of the model analysis.

Résultat de l'analyse des modèles

* if the test filter does not destroy the invariance properties. 